\section{Caracterização dos acidentes escorpiônicos em Belo Horizonte, Minas Gerais, Brasil, 2005 a 2009}

\author{
Epidemiological study of scorpion stings in Belo \\ Horizonte, Minas Gerais State, Brazil, 2005-2009
}

\author{
1 Escola de Veterinária, \\ Universidade Federal de \\ Minas Gerais, Belo Horizonte, \\ Brasil. \\ 2 Hospital João XXIII, \\ Fundação Hospitalar do \\ Estado de Minas Gerais, Belo \\ Horizonte, Brasil. \\ 3 Faculdade de Filosofia \\ e Ciências Humanas, \\ Universidade Federal de \\ Minas Gerais, Belo Horizonte, \\ Brasil. \\ 4 Secretaria Municipal de \\ Saúde de Belo Horizonte, \\ Belo Horizonte, Brasil. \\ Correspondência \\ A. D. Barbosa \\ Escola de Veterinária, \\ Universidade Federal de \\ Minas Gerais. \\ Av. Antonio Carlos 6627, \\ Belo Horizonte, $M G$ \\ 31270-901, Brasil. \\ amandaduarte@ufmg.br
}

\begin{abstract}
This retrospective epidemiological study focused on scorpion stings in Belo Horizonte, Minas Gerais State, Brazil, from 2005 to 2009. Data on reported scorpion stings were taken from the $\mathrm{Na}$ tional System for Notifiable Diseases (SINAN) and the Epidemiological Surveillance System (SISVE) and patient records from the João XXIII Hospital. There were 2, 769 cases of scorpion stings in Belo Horizonte (114.7 cases per 100,000 inhabitants), with a downward trend over time and the highest incidence from August to January. Tityus serrulatus was the species involved in most of the stings. There was no statistical difference in the incidence between male and female victims, and the most frequently affected age bracket was 55 to 64 years. Ninety-six percent of cases evolved to cure, and there were two deaths. It is necessary to improve the reporting process for scorpion stings and to consider the determinants of scorpion stings in order to plan and implement effective public health interventions.
\end{abstract}

Scorpions; Notice; Accidents

\author{
Amanda Duarte Barbosa ${ }^{1}$ \\ Danielle Ferreira de Magalhães 1 \\ José Ailton da Silva 1 \\ Marcos Xavier Silva 1 \\ Maria de Fátima Eyer Cabral Cardoso 2 \\ José Newton Coelho Meneses 3 \\ Maria da Consolação Magalhães Cunha ${ }^{4}$
}

Os escorpiões são artrópodes que podem causar com sua picada um quadro de envenenamento humano cuja gravidade e evolução podem variar amplamente, havendo casos de morte ou de sequelas temporárias ao trabalho ${ }^{1}$. A espécie Tityus serrulatus é a que possui o veneno mais potente e que está mais bem adaptada à vida domiciliar urbana, sendo resistente aos produtos químicos disponíveis para o seu controle 2 . Em 2009, foram notificados no Brasil 45.721 acidentes causados por escorpiões, representando um aumento superior a 7 mil casos, quando comparado ao ano anterior (38.671) 3. No Município de Belo Horizonte, Minas Gerais, de 1990 a 1997, ocorreram 3.265 acidentes; todos eles foram causados por escorpiões amarelos (T. serrulatus) ${ }^{4}$. Em 2004, Belo Horizonte apresentou alta incidência de escorpionismo, representando $66,5 \%$ dos casos atendidos no Serviço de Toxicologia de Minas Gerais do Hospital João XXIII, da Fundação Hospitalar do Estado de Minas Gerais (FHEMIG) 5.

O escorpionismo deve ser objeto constante de ações públicas, visando principalmente ao maior esclarecimento e à mudança de comportamento da população. Para isso, é de alta relevância o conhecimento da epidemiologia do agravo.

Objetivou-se caracterizar os acidentes escorpiônicos no Município de Belo Horizonte, de 2005 a 2009, de acordo com a incidência, distribuição temporal e perfil dos acidentes, dos indivíduos acidentados, dos atendimentos médicos e da evolução dos casos clínicos. 
Foi realizado um estudo epidemiológico observacional retrospectivo baseado nas fontes de dados de notificação de acidentes escorpiônicos do Sistema de Informação de Agravos de Notificação (SINAN) e Sistema de Vigilância Epidemiológica (SISVE) (1.009 casos), ambos de posse da Gerência de Epidemiologia e Informação da Secretaria Municipal de Saúde de Belo Horizonte (SMSA/BH) e compostos de dados coletados na Unidade de Toxicologia do Hospital João XXIII, referentes aos anos 2008 e 2009. Foram também recuperados dados das fichas de atendimento a vítimas de acidentes por escorpião da Unidade de Toxicologia do Hospital João XXIII (1.760 fichas), referentes ao período de 2005 a 2007, até então não digitalizadas. As diferenças de frequências referentes aos acidentes, indivíduos acometidos e atendimentos no período estudado foram analisadas pelo teste do qui-quadrado ${ }^{6}$.

Todas as variáveis utilizadas no estudo estavam presentes em ambas as fontes de dados analisadas, exceto a espécie do escorpião responsável pela picada, presente apenas nas fichas de atendimento clínico do Hospital João XXIII, nas quais existe um campo específico para registro de tal informação. A identificação do escorpião é realizada por um biólogo responsável pelo tombamento dos animais peçonhentos levados ao hospital pelos pacientes no momento do atendimento.

Esta pesquisa foi feita em concordância com os comitês de ética em pesquisa da SMSA/BH, da Universidade Federal de Minas Gerais e da FHEMIG.

Entre 2005 e 2009, ocorreram em Belo Horizonte 2.769 casos de acidentes por escorpião (incidência acumulada no período de 114,7 casos por 100 mil habitantes) (Tabela 1). As incidências anuais verificadas para o município foram maiores do que a incidência verificada por Campolina 5 no ano de 2004 (5,02 casos/100 mil habitan-

Tabela 1

Distribuição anual e incidência, por 100.000 habitantes, dos acidentes escorpiônicos ocorridos em Belo Horizonte, Minas Gerais, Brasil, entre 2005 e 2009.

\begin{tabular}{lccc}
\hline Ano & \multicolumn{2}{c}{ Casos } \\
& $\mathbf{n}$ & $\%$ & Por 100.000 habitantes \\
\hline 2005 & 679 & 24,52 & 28,6 \\
2006 & 566 & 20,44 & 23,6 \\
2007 & 515 & 18,60 & 21,3 \\
2008 & 412 & 14,88 & 16,9 \\
2009 & 597 & 21,56 & 24,3 \\
Total & 2,769 & 100,00 & 114,7 \\
\hline
\end{tabular}

tes). Essas informações confirmam a importância do escorpionismo no Estado de Minas Gerais e no Município de Belo Horizonte como real problema de saúde pública.

Analisando-se o comportamento temporal do agravo, encontrou-se uma tendência decrescente entre os anos de 2005 e 2009 (Figura 1). Esse resultado difere da tendência à estabilidade observada por Nunes et al. 7 e do aumento do número de casos em relação aos anos anteriores mencionado pelo Manual de Diagnóstico e Tratamento de Acidentes por Animais Peçonhentos 8 e em outros estudos 4,5. Enquanto esses autores demonstraram uma melhoria da qualidade da informação, especialmente após a implantação do SINAN no país, em 1988, é provável que em Belo Horizonte esteja opostamente ocorrendo uma deficiência no processo de notificação das informações referentes aos acidentes por escorpião. Isso pode ser evidenciado pelo fato de ter havido a necessidade de resgate de dados em fichas clínicas do Hospital João XXIII referente aos anos de 2005 a 2007. Não se sabe, então, se as notificações dos anos 2008 e 2009 refletem a situação real do agravo, ou seja, até que ponto houve melhorias no fluxo de informações. Apesar das evidências de subnotificação com base na tendência decrescente de casos no período, deve-se considerar também a hipótese de melhorias no controle realizado pelo serviço público.

Em relação à distribuição mensal, o maior percentual de casos ocorreu entre os meses de setembro a janeiro. A ocorrência de escorpionismo é maior nos períodos mais quentes e chuvosos do ano, particularmente pelo desalojamento de escorpiões e saída desses animais para a caça por alimentos. Distribuição semelhante de sazonalidade foi verificada na Região Sudeste do Brasil 3,8.

Em relação à espécie do escorpião, verificouse que em apenas $39 \%$ do total de casos notificados esta informação foi registrada. No caso das fichas clínicas do Hospital João XXIII, o não registro da informação pode estar relacionado ao fato de o paciente não ter levado o escorpião para identificação no momento do atendimento ou mesmo ao preenchimento incompleto dos prontuários, fato observado por Campolina 5. Nas fichas do SINAN/SISVE, como já mencionado, não consta um campo específico para registro da espécie do escorpião. Dentre os casos notificados em que a espécie do escorpião foi identificada, a maioria $(99,42 \%)$ teve o T. serrulatus como responsável pelo acidente. Isso prova a grande adaptação desta espécie ao meio urbano e o risco que ela representa para a saúde pública, como verificado por outros autores 4,5,9. A espécie $T i$ tyus bahiensis foi identificada em apenas 4 casos, 
Tendência do número de casos de acidente escorpiônico, por 100.000 habitantes, em Belo Horizonte, Minas Gerais, Brasil, entre 2005 e 2009.

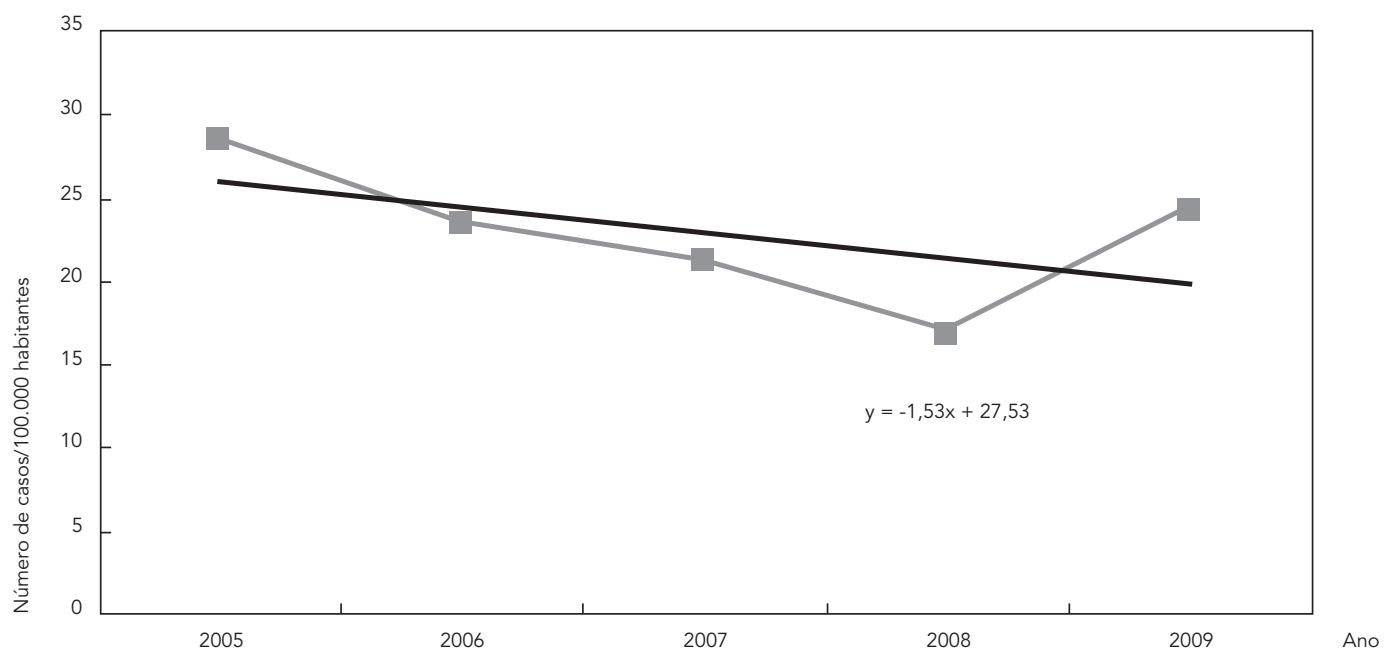

representando 0,58\% das notificações em que houve identificação da espécie.

O ambiente de exposição mais frequente foi o residencial (59\%), seguido pelo ambiente de trabalho (11\%), ambiente externo (6\%) e ambiente escolar $(0,28 \%)$. O "ambiente externo" se refere às ruas e praças públicas, cemitérios, supermercados, dentre outros. Em $24 \%$ dos casos o ambiente de exposição não foi notificado. As atividades domésticas podem constituir fator de risco importante no caso desse agravo, o que foi verificado também por Campolina 5 .

Dentre os locais do corpo onde os indivíduos sofreram a picada do escorpião, os mais frequentes foram mãos e pés $(24,76 \%$ e $20,37 \%$, respectivamente), seguidos por coxa $(15,46 \%)$, tronco $(15,45 \%)$, perna $(4,84 \%)$, braço $(4,23 \%)$, antebraço $(3,29 \%)$ e cabeça e pescoço $(2,28 \%)$. Em $9,32 \%$ dos casos, o local da picada não foi notificado. Resultados semelhantes foram verificados por Nunes et al. 7 e Campolina 5 e ressaltam o risco de se manipular objetos e de pisar no chão ou calçar sapatos sem prévia observação.

Não foi encontrada diferença estatística ( $\mathrm{p}>$ 0,05 ) entre os indivíduos acidentados em relação ao gênero, o que corrobora as observações de Nunes et al. 7 e registros do SINAN (http://tabnet. datasus.gov.br, acessado em 20/Set/2010).

A análise da taxa de incidência acumulada de casos para o período de 2005 a 2009, de acordo com a faixa etária da população vítima da picada, mostrou que indivíduos de 55 a 64 anos apresentaram maior risco para o escorpionismo (158,6 casos/100 mil habitantes), assim como foi constatado por Nunes et al. 7 . Em 1,88\% dos casos a idade não foi notificada.

Em relação ao tempo entre a picada e o atendimento, categorizado por intervalo de "0-1 hora" e "> 1 hora", verificou-se diferença estatisticamente significativa entre as frequências registradas $(\mathrm{p}<0,05)$. Tal análise mostra que a maioria dos casos no período, dentre os que tiveram a variável em questão notificada, foi atendida em caráter de urgência (até uma hora), o que difere dos resultados de Campolina 5. Do ponto de vista da vigilância epidemiológica, essa informação é importante, pois pode significar melhoria da qualidade da informação à população no que se refere à necessidade da procura pelo serviço médico em caráter de urgência no caso de picada por escorpião. A urgência dos atendimentos é de extrema importância uma vez que um possível quadro clínico sistêmico pode se estabelecer no paciente de alguns minutos a poucas horas 8 .

Em todo o período, a manifestação clínica mais constante foi a local $(86,2 \%)$, a qual caracteriza um quadro clínico leve de escorpionismo. Apenas 8,99\% das vítimas apresentaram algum sintoma sistêmico, com quadro clínico moderado ou grave. Observou-se ainda que $1,85 \%$ dos 
acidentados que recebeu atendimento médico não manifestou nenhum sintoma e em 2,96\% dos casos o tipo de manifestação clínica não foi notificada.

Na maioria dos casos $(91,04 \%)$ não foi necessária soroterapia, que foi utilizada em apenas $7,73 \%$ dos casos. Não houve notificação relacionada a essa variável em 1,23\% dos casos. Esse resultado condiz com as porcentagens referentes às manifestações clínicas descritas anteriormente, uma vez que a soroterapia não deve ser adotada nos casos leves, vale dizer, na ausência de manifestações sistêmicas 2 .

Em relação à evolução do quadro clínico, 95,9\% dos casos receberam alta hospitalar.

\section{Resumo}

Realizou-se um estudo epidemiológico observacional retrospectivo para caracterizar os acidentes escorpiônicos em Belo Horizonte, Minas Gerais, Brasil, entre 2005 e 2009. Foram coletados dados do Sistema Nacional de Informação de Agravos de Notificação (SINAN) e Sistema de Vigilância Epidemiológica (SISVE) e das fichas de atendimento do Hospital João XXIII. Observou-se um total de 2.769 casos no período $(114,7 / 100$ mil habitantes), tendência decrescente e maior ocorrência entre agosto e janeiro. A espécie Tityus serrulatus foi responsável pela maioria das picadas. Não houve diferença estatística entre os gêneros dos indivíduos acometidos e a faixa etária entre 55 e 64 anos foi a que apresentou maior risco para escorpionismo. Noventa e seis por cento dos quadros clínicos evoluíram para a cura, tendo sido observados dois óbitos. Alerta-se para necessidade de melhorias no processo de notificação de acidentes por escorpião e sugere-se considerar seus determinantes para o planejamento e direcionamento de intervenções por parte do serviço público de saúde.

Escorpiões; Notificação; Acidente
Ocorreram dois óbitos em crianças de 4 e 6 anos, respectivamente, sendo um em 2005 e outro em 2009. A taxa de letalidade para o período foi de $0,07 \%$. Tipo de evolução não notificado representou $2,74 \%$ do total.

Os resultados referentes à gravidade, tratamento dos casos e evolução estão de acordo com os obtidos por Cupo et al. ${ }^{9}$, Campolina 5 e com registros do SINAN (http://tabnet.datasus.gov. br, acessado em 20/Set/2010), que mostraram a predominância de casos leves que evoluem para alta hospitalar, para a maioria dos quais não foi utilizada soroterapia como tratamento.

\section{Colaboradores}

A. D. Barbosa dedicou-se à elaboração do projeto de pesquisa, levantamento bibliográfico, resgate, organização e análise dos bancos de dados; elaborou e redigiu a introdução, metodologia, resultados, discussão e conclusões; e participou da revisão final. D. F. Magalhães auxiliou na elaboração da metodologia da pesquisa e na redação dos resultados, discussão e conclusões e participou da revisão final do texto. J. A. Silva participou da etapa de elaboração do projeto de pesquisa e da discussão e conclusões do trabalho, bem como da revisão final do texto. M. X. Silva colaborou com a análise estatística dos resultados e com a revisão final do texto. M. F. E. C. Cardoso colaborou com a etapa de coleta de dados e com a revisão final do texto. J. N. C. Meneses e M. C. M. Cunha contribuíram com a elaboração do projeto de pesquisa e obtenção dos bancos de dados utilizados, bem como com a revisão final do texto.

\section{Agradecimentos}

À Fundação de Amparo à Pesquisa do Estado de Minas Gerais (FAPEMIG) e Coordenação de Aperfeiçoamento de Pessoal de Nível Superior (CAPES). 


\section{Referências}

1. Ministério da Saúde. Vigilância em saúde - zoonoses. Brasília: Ministério da Saúde; 2009. (Série B. Textos Básicos de Saúde) (Cadernos de Atenção Básica, 22).

2. Ministério da Saúde. Manual de controle de escorpiões. Brasília: Ministério da Saúde; 2009.

3. Ministério da Saúde. Situação epidemiológica das zoonoses de interesse para a saúde pública. Boletim Eletrônico Epidemiológico 2010; 10(2).

4. Soares MRM, Azevedo CSMM. Escorpionismo em Belo Horizonte, MG: um estudo retrospectivo. Rev Soc Bras Med Trop 2002; 35:359-63.

5. Campolina D. Georreferenciamento e estudo clínico-epidemiológico dos acidentes escorpiônicos atendidos em Belo Horizonte, no serviço de toxicologia de Minas Gerais [Dissertação de Mestrado]. Belo Horizonte: Universidade Federal de Minas Gerais; 2006.
6. Sampaio IBM. Estatística aplicada à experimentação animal. Belo Horizonte: Fundação de Estudo e Pesquisa em Medicina Veterinária e Zootecnia; 1998.

7. Nunes CS, Bevilacqua PD, Jardim CCG. Aspectos demográficos e espaciais dos acidentes escorpiônicos no Distrito Sanitário Noroeste, Município de Belo Horizonte, Minas Gerais, 1993 a 1996. Cad Saúde Pública 2000; 16:213-23.

8. Fundação Nacional de Saúde. Manual de diagnóstico e tratamento de acidentes por animais peçonhentos. Brasília: Fundação Nacional de Saúde; 2001.

9. Cupo P, Azevedo-Marques MM, Hering SE. Acidentes por animais peçonhentos: escorpiões e aranhas. Medicina (Ribeirão Preto) 2003; 36:490-7.

Recebido em 14/Dez/2011

Versão final reapresentada em 16/Mai/2012

Aprovado em 13/Jun/2012 